\title{
Intelligent automation and IT for the optimization of renewable energy and wastewater treatment processes
}

\author{
Michael Bongards, Daniel Gaida, Oliver Trauer and Christian Wolf
}

\begin{abstract}
Background: Environmental systems often have a very complex structure. Methods from computational intelligence $(\mathrm{Cl})$ that are often inspired by nature can help to improve these systems. On the one hand, $\mathrm{Cl}$ methods can be used for optimization; on the other hand, they can be used to extract information out of time series recorded from environmental systems.
\end{abstract}

Methods: Methods from different fields of computational intelligence are investigated. Among them are supervised and unsupervised machine learning methods used for classification and cluster analysis, respectively. Furthermore, methods from evolutionary computation and multi-agent systems are used to develop control and optimization solutions for environmental processes.

Results: In this paper, five applications in the fields of anaerobic digestion, pellet-heating, and wastewater management are studied. Using Cl methods, e.g., biogas plant operation or a pellet-heating process can be optimized. Furthermore, important process variables can be obtained from huge measurement datasets that otherwise would be unanalyzed and therefore data cemeteries.

Conclusions: The results reveal that using $\mathrm{Cl}$ methods environmental processes can be improved in a favorable cost-benefit fashion.

Keywords: Biogas; Environmental engineering; Evolutionary computation; Image analysis; Machine learning; Multi-agent systems

\section{Background}

The future success of renewable energy as an alternative energy source to fossil fuels is an essential part of the German future vision of the 'Energiewende' (energy transition). It will be fostered by two independent aspects: (1) political regulations and (2) advantage over classical energy sources in price competition. Because aspect (1) is situated more in the area of social sciences and is, in many ways, a more stochastic process, the following ideas will focus on aspect (2).

At the intersection of classical automation and more sophisticated computational intelligence (CI) methods, there is a huge potential to develop intelligent automation systems. Applied to renewable energy processes and

\footnotetext{
* Correspondence: michael.bongards@fh-koeln.de

Cologne University of Applied Sciences, Steinmueller Allee 1, 51643 Gummersbach, Germany
}

plants, those will be strengthened in terms of efficiency, stability, and financial viability, increasing their potential of long-term success. The goal is to improve system performance to an extent such that the initial investment in expertise and infrastructure is paid out in the long run. As renewable energies depend on natural forces, which are complex and difficult to control, finding optimal solutions is challenging. CI methods, which are often inspired by natural processes and phenomena, offer an interesting opportunity to find optimal solutions for specific applications. As CI is a wide field, the focus of this paper will be on the following:

(1) Supervised machine learning

(2) Unsupervised machine learning

(3) Evolutionary computation

(4)Multi-agent systems 
They are preceded by an application of a standard data analysis task which is not based on CI methods. All methods are applied to one of the following environmental, renewable applications:

(1)Anaerobic digestion in biogas plants

(2) Pellet-heating process

(3) Wastewater management and treatment

An overview of artificial intelligence techniques applied to environmental systems in general can be found in [1], to photovoltaic systems in [2], and to smart electric grids in [3].

The possibilities for continuous process improvements by intelligent automation and data-analysis methods will be shown by five typical examples that are under development or test in our research group GECO $\mathrm{C}^{\mathrm{a}}$ :

(1) By elaborated benchmarking-technologies, the efficiency of biogas plants can be improved. (2) Intelligent data analysis enables the application of innovative measurement technologies to allow for comprehensive monitoring and stability improvement of biogas plants. (3) The dynamic simulation of the fermentation process opens the way to further optimization of the biogas process. (4) The application of enhanced algorithms allows the use of image-processing technologies to control and optimize incineration processes, and software agents (5) enable the control of highly distributed systems, i.e., flexible energy production and distribution within smart grids.

The background of those five applications and the used methods are introduced in the 'Methods' section and results shown and discussed in the last section.

\section{Methods}

Using standard statistics, such as mean, standard deviation as well as correlation and linear regression analysis, efficiency of processes in terms of input-output relations, and linear dependencies in the data, can be detected and evaluated. In the following application, those methods are used to evaluate as new substrate pretreatment process at a full-scale agricultural biogas plant. To properly asses biogas plant performance and optimization potential, detailed regular data analysis is a key factor. Benchmarking allows the comparison of innovative feed-treatment technologies. The effects of substrate pretreatment on biogas yield and substrate degradation by mixing and cutting at an agricultural biogas plant (ABP) were closely investigated. The purpose of the test is to identify the benefits of the changed physical structure of the substrates on ABP operation. The theory is that the physical structure of the substrate feed after treatment improves overall feed efficiency (net yield of useful outputs per unit input) of biogas plants. For a review, see [4].
At a full-scale industrial biogas plant, supervised machine learning methods are applied to measure organic acid concentration in the digesters. Being common knowledge that organic acids, volatile fatty acids (VFA) in particular, are crucial to assess process stability, their online measurement allows for the development and implementation of new advanced control strategies for anaerobic digestion processes. Modern spectroscopic online measurements allow for online detection of organic acids using ultraviolet/visible (UV/vis) and middle infrared (MIR) spectrometric probes. The close monitoring of anaerobic digestion processes and the development of control strategies for optimal organic acid concentrations will substantially increase process efficiency and stability. As methods, different classification and regression algorithms are used such as discriminant analysis [5], support vector machines, and artificial neural networks. Using a spectrometric probe measuring in the middle infrared spectral range, it is possible to measure total organic carbon and ammonium as well. An application to an industrial biogas plant within the metabolon project $^{\mathrm{b}}$ is presented in the results section. Supervised machine learning algorithms are often applied to environmental systems, e.g. [6-8]. A review of their application to environmental process data is given in [9].

The unsupervised machine learning method $k$-means clustering [10] was used to analyze the flame of a pelletheating process. The increasing demand in future $\mathrm{CO}_{2}$ neutral heat generation by modern biomass pellet heaters leads to increasing demands for these pellet heaters and to a higher demand for suitable fuels. Traditional fuels such as wood chips are replaced by different mixed fuels made of recyclables. This leads to new challenges for existing classic control and monitoring systems because every new fuel has different burn characteristics (depending on density, humidity, composition, dosage). The applied control strategy in today's biomass heater industry uses exclusively static control parameters with the use of sensor based measurement systems. The corresponding control parameters are set by experienced service staff at the commissioning of the plant for the use of a specific fuel type. In most instances, the optimal setting of the control parameters is done by a visual assessment of the combustion process. In the case that the plant operator changes the type of fuel, these control parameters need to be adapted to the new fuel resulting in high service costs for the operator and long adaptation times until an optimal control is reached. Since these parameters can only be manually tuned in, a quick adjustment is not possible. One of the most important control objectives of a combustion process of pellet heaters is optimal air and pellet supply to ensure energy-efficient, clean, and pollution-minimized combustion. The most common control parameter is temperature, measured in the pumice stone coating of 
the boiler. Due to its location, the temperature measurement suffers from long dead times, which is a disadvantage for time-sensitive control strategies. Sometimes, a pollution sensor at the off-gas complements the temperature sensor but like the temperature sensor, it is an indirect measurement of the combustion quality which requires further analysis. The application of digital image processing offers the advantage of direct and immediate measurement of combustion process quality. Time-sensitive control strategies can then be used to optimize the process. Various statements about the quality of a combustion process can be done just by observing color and surface of the flame. A large flame surface usually indicates a low excess air rate and a flame color which is mostly red or a flame with dark spots usually indicate a high concentration of carbon monoxide as well as increased soot. An optimal burn with low pollutant and high efficiency is recognized by semi-transparent large yellow flames whereas small flame surfaces indicate low efficiency and high emissions. The combination of modern image processing and cluster algorithms offers the ability to distinguish between the different properties of the flame.

To optimize and control the substrate feed of a biogas plant, evolutionary computation methods are applied to find the optimal feed with respect to a complex objective function. Detailed simulation models of the anaerobic digestion process, such as the IWA Anaerobic Digestion Model No. 1 (ADM1) [11], are valuable platforms for developing and testing new optimization and control strategies. The ADM1 is a structured model incorporating disintegration and hydrolysis, acidogenesis, acetogenesis, and methanogenesis steps. Therefore, it offers the complexity needed to model full-scale biogas plants realistically. The availability of a validated simulation model allows powerful computational intelligence methods such as particle swarm optimization (PSO) [12] and genetic algorithms (GA) [13] to be used to estimate optimal operating parameters for the biogas plant. Nevertheless, application of these powerful population based optimization procedures is only feasible with a simulation model because testing of many different operating parameters at full-scale biogas plants is difficult and often not practical. For example, variations in substrate feed can affect process stability and cause extreme situations that are difficult to recover from.

To control the level of storm water retention tanks, an approach is used which belongs to the field of multi-agent systems. Such software agent concepts are developed in the research area of distributed artificial intelligence. The origins date back to the 1950s of the last century. A development leap of the agent technology happened in the age of massive computer networks and increasing complexity of distributed applications. Software agents are nowadays used in many applications in business, industry, and environment, e.g. [14-18]. They often assist the user in the background, without him realizing it. Often, an agent is viewed as an autonomous unit which can perform assigned tasks in a defined environment while the agent perceives its environment through sensors. On the basis of its perception, it decides which measures he can perform in order to achieve his goals. Through his actuators, it influences the environment.

\section{Results and discussion}

This section is divided into five subsections, each one discussing the results of one application introduced in the previous section.

\section{Benchmarking of feed optimization in biogas plants}

To test the effects of an optimized substrate feed mix, a commercial pretreatment unit is used to feed an ABP near Gummersbach for a period of 19 weeks from 1 May 2012 till 3 September 2012.

To evaluate the efficiency of the pretreatment, the trial was split into three different phases following an initial phase of nearly constant substrate feeding to allow for a good assessment of the start state of the ABG. Thus, the different phases of the trial are as follows:

- Before trial: 1 May 2012 to 16 July 2012 ○ Ø Substrate feed: $19 \mathrm{t}$ maize, $27 \mathrm{~m}^{3}$ manure, and 1.5 t dung

- First stage: 17 July 2012 to 30 July 2012 (only maize pretreated)

$\circ \varnothing$ Substrate feed: $19.5 \mathrm{t}$ maize, $23 \mathrm{~m}^{3}$ manure, and 2 t dung

- Second stage: 31 July 2012 to 13 August 2012 (maize and manure mixed and pretreated)

$\circ \varnothing$ Substrate feed: $19.5 \mathrm{t}$ maize, $23 \mathrm{~m}^{3}$ manure, and 2 t dung

- Third stage: 14 August 2012 to 3 September 2012 (maize, manure, dung, and straw mixed and pretreated)

○ Ø Substrate feed: $19.7 \mathrm{t}$ maize, $26 \mathrm{~m}^{3}$ manure, $1.7 \mathrm{t}$ dung, 1 bale of straw

The overall goal is to compare ABP operation before, during the trial, and in each of three trial stages, respectively. Therefore, the phase before the trial, the whole trial period, and the three stages are analyzed separately and then compared, which allows a performance evaluation of each trial stage as well as of the trial as a whole. The following statistical and anaerobic-typical parameters are calculated and performed:

- Mean and standard deviation of all recorded parameters during the different phases

- Biogas and power production 
- Specific biogas production $\left(\mathrm{m}^{3} / \mathrm{t}\right.$ of substrate)

- Specific biogas production $\left(\mathrm{m}^{3} / \mathrm{m}^{3}\right.$ digester volume)

- Specific power production ( $\mathrm{kW} / \mathrm{t}$ of substrate)

- Specific power production $\left(\mathrm{kW} / \mathrm{m}^{3}\right.$ digester volume $)$

- Hydraulic retention time (d)

Based on these parameters, the improvement during each phase of the trial compared to the state of the ABG before the trial is calculated in percent [\%]. For better visualization of analysis results, bar plots as well as trajectories over time are used.

Figure 1 shows that a significant reduction in standard deviation of the biogas production was achieved throughout the whole trial. This shows that the AD process is more stable and efficient.

The specific power production per ton of substrate $[\mathrm{kW} / \mathrm{t}]$ shows similar results as the specific biogas production which is expected as these parameters are closely related; see Figure 2. In general, an increase in specific power production is evident although stage 3 of the trial performs again worse than the other stages of the trial. Nevertheless, overall increase in specific power production is significant with up to $9 \%$ in stage 1 and $6.8 \%$ in average over the whole trial period when compared to the specific power production before the trial.

Overall, the results of the data analysis clearly show that the use of a pretreatment process for the substrate feed of biogas plants is beneficial to process efficiency. During the trial stages, an improvement with a view to specific biogas and power production between $4 \%$ in the worst case and $14 \%$ in the best case was achieved. In average, an improvement of $7 \%$ for the specific biogas production $\left(\mathrm{m}^{3} / \mathrm{m}^{3}\right.$ digester volume) and of $12 \%$ for the specific power production $\left(\mathrm{kW} / \mathrm{m}^{3}\right.$ digester volume) was proven by the data analysis. Furthermore, an analysis of the standard deviation of biogas and power production throughout the whole trial in comparison to the standard deviation before the trial shows a decrease in standard deviation of $48.5 \%$ for biogas production and of $8.7 \%$ for power production. This clearly indicates a significant improvement in process stability during plant operation caused by a homogenized substrate feed. Besides all
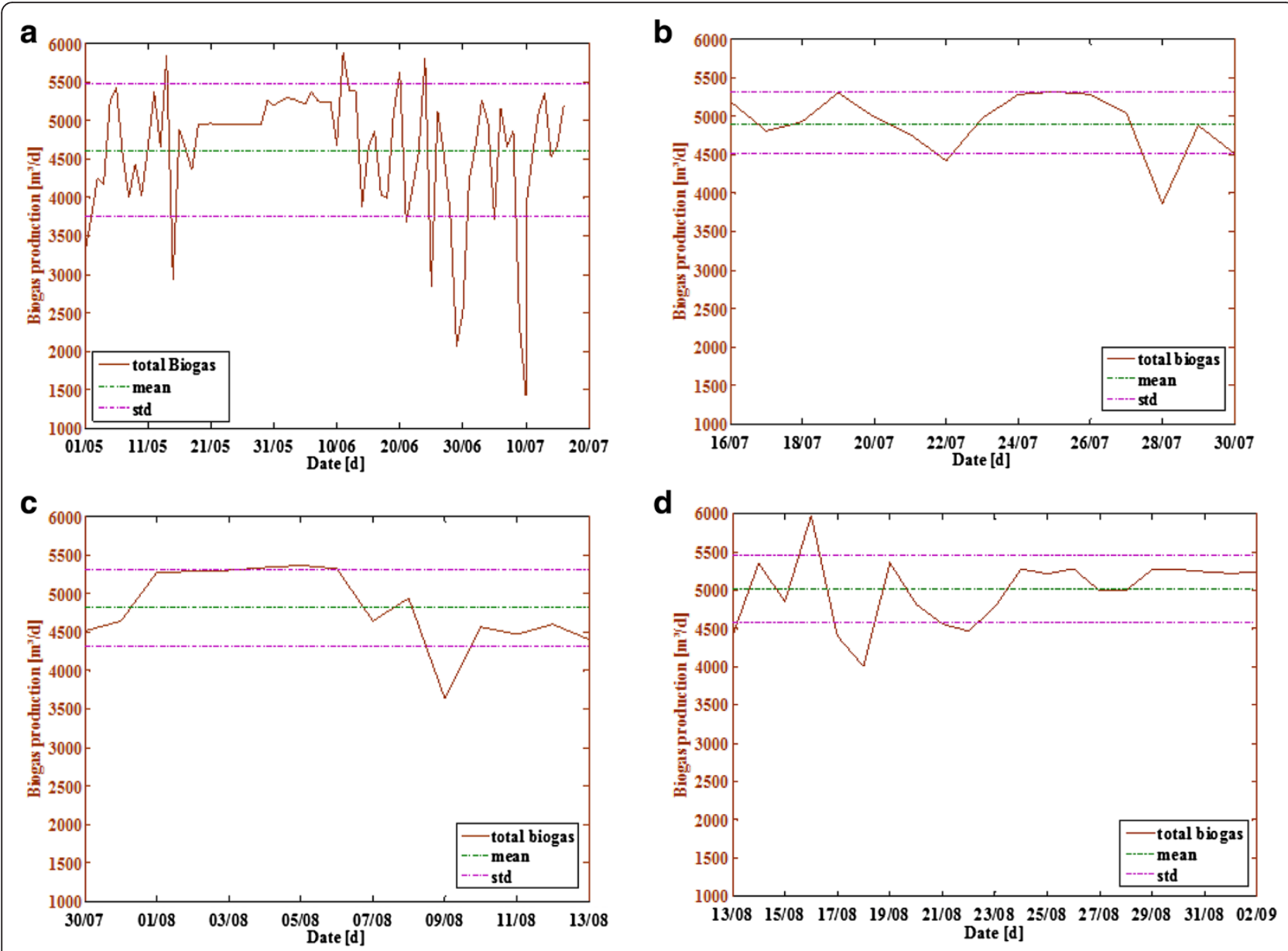

Figure 1 Biogas production. Comparison of Biogas production $\left[\mathrm{m}^{3} /\right.$ day] before the trial (a) and during first (b), second (c), and third (d) phase of the trial. 
a) mecific power production per $\mathrm{t}$ of substrate $(\mathrm{kW} / \mathrm{t})$

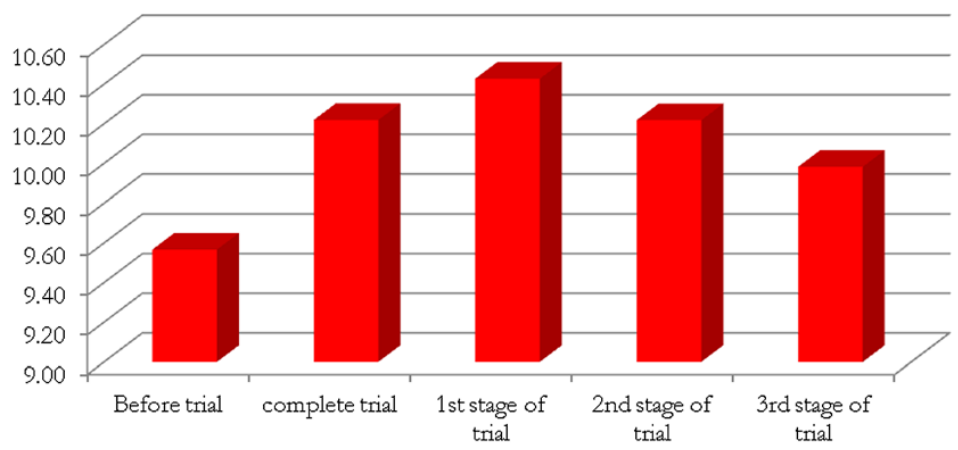

b)

- increase in specific power production (\%)

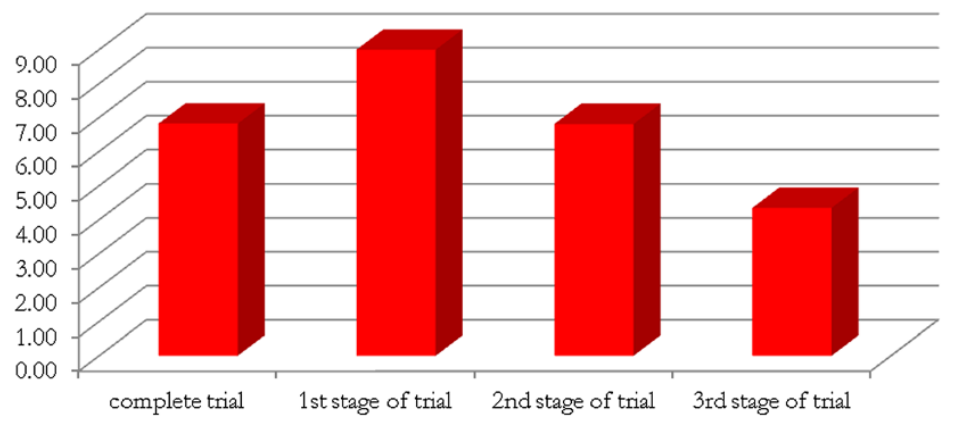

Figure 2 Specific power production. (a) Comparison of specific power production per $t$ of substrate [kW/t] compared to the state before trial and the three stages of trial. (b) Comparison of increase in specific power production in percent [\%] also compared to the state before trial and the three stages of trial.

these positive results, a clear improvement through the use of straw in the pretreatment process could not be proven during trial. Figure 2 shows that although an improvement is achieved by using straw in stage 3 , it is significantly lower than for stages 1 and 2 .

\section{Intelligent data analysis as part of innovative measurements for optimization in biogas plants}

Online monitoring of process variables that are crucial to stable and effective biogas production at agricultural and industrial biogas plants such as volatile fatty acids (VFAs), carbon buffer (TA), and ammonium concentration $(\mathrm{NH} 4+)$ is the basis for each kind of process optimization and control. Unfortunately, these variables are difficult to measure online due to high total solids concentrations (up to $15 \%$ ) and sensor drifts caused by clogging and fouling of the probes. The solutions to this problem are spectroscopic measurement systems, which measure absorption or reflection spectra which are subsequently analyzed and translated into process variable concentrations. Due to the fact that the relationship between spectral data and actual variable concentration is far from linear and sometimes difficult to determine, intelligent data analysis is key to obtain valuable process information. The use of machine learning algorithms has proven to be very useful for this application. In the following two sections, the results from an ultraviolet/visible (UV/vis) and middle infrared (MIR) spectroscopic probe show that VFA and other important process variables can be measured online successfully.

\section{UV/vis spectroscopic probe}

The measurement data set was created from online measurements using the UV/vis measurement system installed at an industrial biogas plant (IBP) and laboratory measurements, which are regularly taken at the IBP to monitor organic acid concentration in the two digesters. Thus, no synthetic data set was used for training and validation of the machine learning methods. Overall, a total of 4,435 samples were obtained from the biogas plant and these were used to generate training and validation data sets with 3,326 and 1,109 samples, respectively. Those samples were subsequently split into five classes containing samples from low VFA concentrations (class 1) up to high VFA concentrations (class 5). To analyze the data in these classes, different data transformation, reduction, and classification methods were used and compared. 
The results prove that this online-measurement is far from trivial, such that advanced pattern recognition methods are needed to achieve good results. This is mainly due to the UV/vis fingerprint which does not show clear absorption peaks. Instead, the absorption patterns of variables are overlapping. Nevertheless, a comparison of the different data transformation, reduction, and classification methods shows that unbalanced data sets for training are a major problem, when it comes to achieving low NMCR (normalized mean misclassification rate) and MCR (mean misclassification rate) results with some classifiers (Table 1). However, application of appropriate class weightings during the training process can effectively counter the effect of the very small set of samples which was available for class 5 .

Of the methods considered, the combination of RF and GerDA yields the best error rate for the unweighted data set $(12.1 \%)$. With regard to the weighted data set, SVM achieves the best overall results with an NMCR of $12 \%$ without requiring any feature selection or extraction methods. The comparison of the weighted SVM and weighted relevance vector machine (RVM) on the GerDA features reveals that both methods perform equally well on the test data set, but that RVMs are more robust and provide more efficient predictive performance due to the significantly lower number of support vectors.

\section{MIR spectroscopic probes}

Using the attenuated total reflectance (ATR) technology and the Fourier-transformed infrared (FTIR) spectroscopy

Table 1 Overall results with NMCR and MCR [19]

\begin{tabular}{llll}
\hline Feature extractor & Classifier & NMCR [\%] & MCR [\%] \\
\hline LDA & Linear & 34.0 & 35.7 \\
GerDA & Linear & 12.8 & 13.1 \\
RF & RF & 16.7 & 17.0 \\
GerDA & RF & 12.1 & 12.4 \\
None & SVM & 19.1 & 10.8 \\
RF & SVM & 19.1 & 10.8 \\
RF & RVM & 25.9 & 15.4 \\
GerDA & RVM & 19.8 & 10.8 \\
FSR & MLP & 30.5 & 16.3 \\
GerDA & MLP & 30.7 & 12.4 \\
None & W-SVM & 12.0 & 12.0 \\
RF & W-SVM & 12.5 & 14.3 \\
GerDA & W-SVM & 12.8 & 11.8 \\
GerDA & W-RVM & 13.0 & 11.0 \\
GerDA & W-MLP & 17.2 & 13.3 \\
FSR & W-MLP & 30.6 & 22.1 \\
\hline
\end{tabular}

in the middle infrared (MIR) spectral range (1,800 to $800 \mathrm{~cm}^{-1}$ ), the process variables VFA, carbon buffer (TA), and $\mathrm{NH} 4+$ can be measured online, simultaneously. The MIR probe and its fully automated process interface for cleaning and calibration (Figure 3) were developed within the research project INNO-MIR BIOGAS ${ }^{\mathrm{c}}$ (development and automation of an innovative MIR online measurement system for biogas plants).

The MIR sensor technology has two major advantages compared to near infrared (NIR) or UV/vis spectroscopic sensors. (1) The important process variables show distinctive peaks in the MIR spectrum which facilitates spectral data analysis. (2) Diamond-tipped ATR probes are extremely robust and low maintenance.

The following figure (Figure 4) shows the relevant wavenumbers for carbon buffer $\left(1,630\right.$ and $\left.1,360 \mathrm{~cm}^{-1}\right)$ and ammonium $\left(1,460 \mathrm{~cm}^{-1}\right)$ (a) and VFA (1,548 and $1,412 \mathrm{~cm}^{-1}$ ) (b).

Using intelligent data analysis methods such as partial least square (PLS) regression and support vector regression (SVR), good calibration models were calculated. The

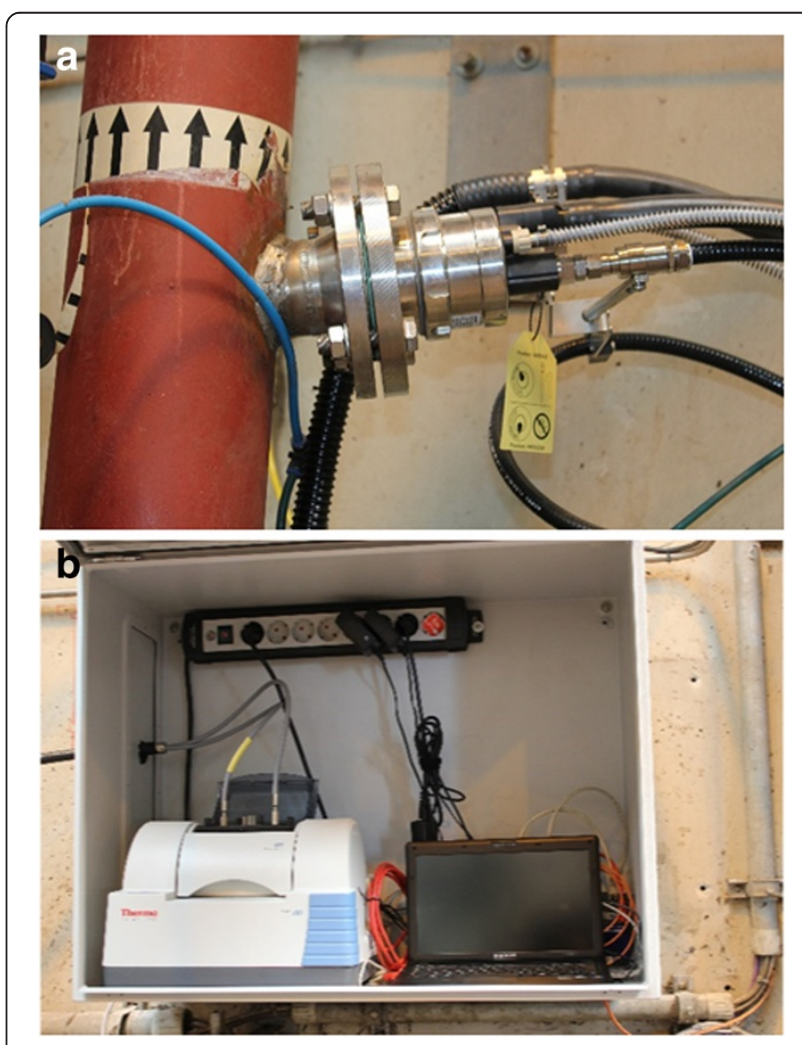

Figure 3 MIR probe installation at an industrial biogas plant. In (a), you can see the probe and probe fitting with cleaning chamber. In (b), the MIR spectrometer and the computer for sensor control are shown. 

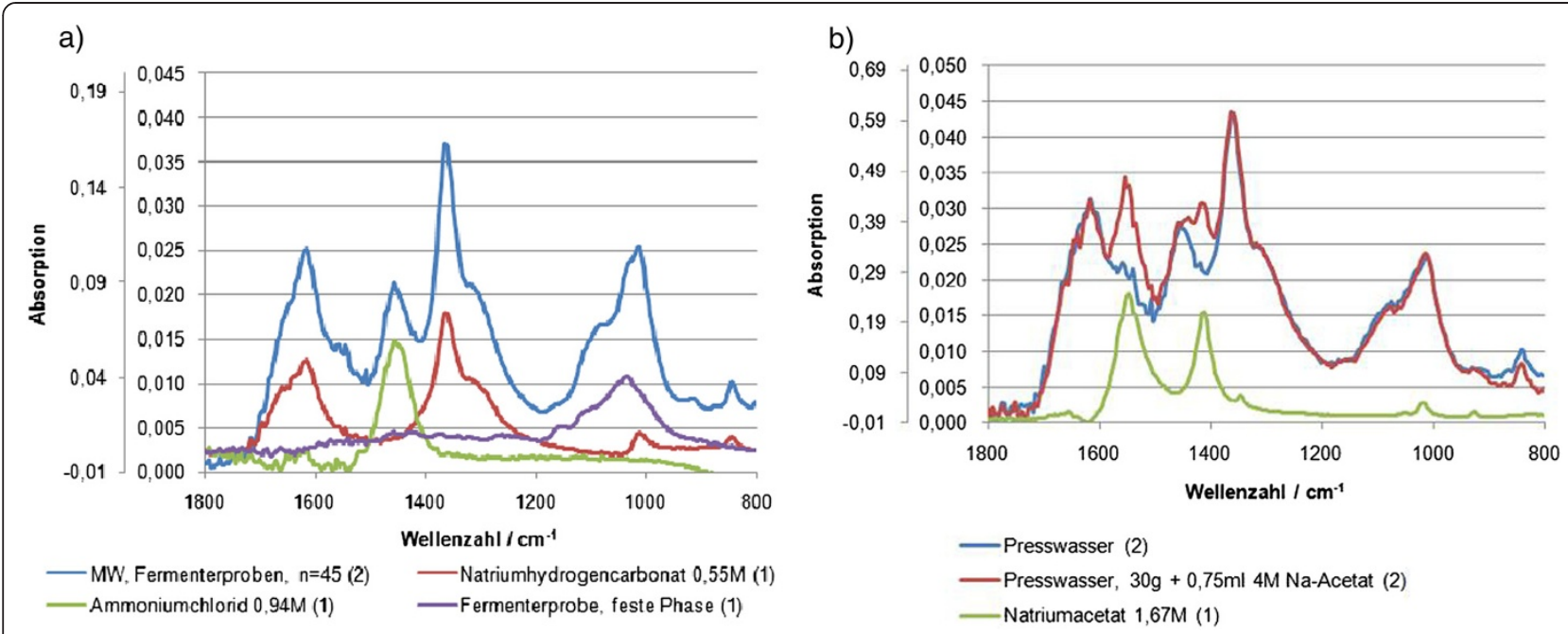

Figure 4 Absorption peaks and relevant wavenumbers for AD process variables. In (a), the wavelengths of ammonium, carbon buffer, and total solids after addition of ammonium chloride and sodium bicarbonate are shown. In (b), VFA-wavelengths after addition of sodium acetate can be seen.

standard errors of cross-validation that were achieved in a controlled laboratory environment are $0.372 \mathrm{~g} / \mathrm{L}$ (VFA: $R^{2}$ $=0.971), 0.336 \mathrm{~g} / \mathrm{L}$ (TAC: $\left.R^{2}=0.996\right)$, and $0.171 \mathrm{~g} / \mathrm{L}\left(\mathrm{NH}_{4^{-}}\right.$ $\left.\mathrm{N}, R^{2}=0.992\right)$ in the case of PLS and 0.386, 0.259, and $0.110 \mathrm{~g} / \mathrm{L}$ for the SVR-algorithm, respectively.

First, in-line measurements in an industrial biogas plant, which uses biowaste as feedstock, show that the expected absorption bands can be found during normal operating conditions. However, they are subject to a temperature effect, which must be taken into account in the quantification. Further studies are needed to put the in-line capability of the measurement system to the test.

\section{Low-cost image analysis for optimization of pellet heating process}

To analyze the flame of a pellet-heating process, the recorded combustion process (see Figure 5) is first converted into a binary image. During this process, the flame portion of the image is set to white (binary value 1 ) and the background is set to black (binary 0). The creation of binary images is carried out by utilizing the method of Otsu [20], which automatically calculates the threshold which is used to decide which pixels will be set to 1 or 0 . By calculating the ratio between the number of white pixels and the total number of pixels of the binary image, the size of the flame is determined. The binary picture is then used for separating the flame from the background of the image by superimposing the binary image with the original image. The resulting (masked) image will then be used for the cluster analysis in the next main step. Before cluster analysis is performed, the image is converted in the device independent $L^{*} \mathrm{a}^{*} \mathrm{~b}$ color space. The basis for this color space is the color opponent theory, where the color and brightness information are in separate channels. The parameter $L^{*}$ represents the luminanceinformation in a range of 0 to 100 whereas parameters $a *$ and $b *$ contain the color information with a range from -100 to 100 . In accordance with the color opponent theory, the green to red color part is represented by $\mathrm{a}^{*}$ and the blue to yellow part by $\mathrm{b} *$. The advantage of this conversion is based on the fact that the color information can be analyzed separately from the brightness information. After the conversion, the $k$-means cluster analysis separates the colors into different clusters. Each cluster is equivalent to one of the three main flame colors (red, yellow, white) that can occur during combustion and represents the different visual properties of the flame. Analyzing those properties and creating different rule sets to identify the combustion state offers the ability to implement low-cost real-time controllers. Nevertheless, the accuracy is strongly affected by fouling caused by soot production. In this case, a controlled air flow into the combustion chamber together with a specially impregnated glass window can be used to minimize soot production.

Simulation of biogas plants for feed optimization and control The optimization of the substrate feed with regard to its flow rate (throughput) and composition is a highly nonlinear and complex optimization problem. CI methods such as genetic algorithms and particle swarm optimization can be used to solve this task. GAs and PSO are both methods that optimize the optimization criterion using exploration and exploitation schemes. This allows a large search space to be explored and can lead to novel solutions that would normally not be considered. 

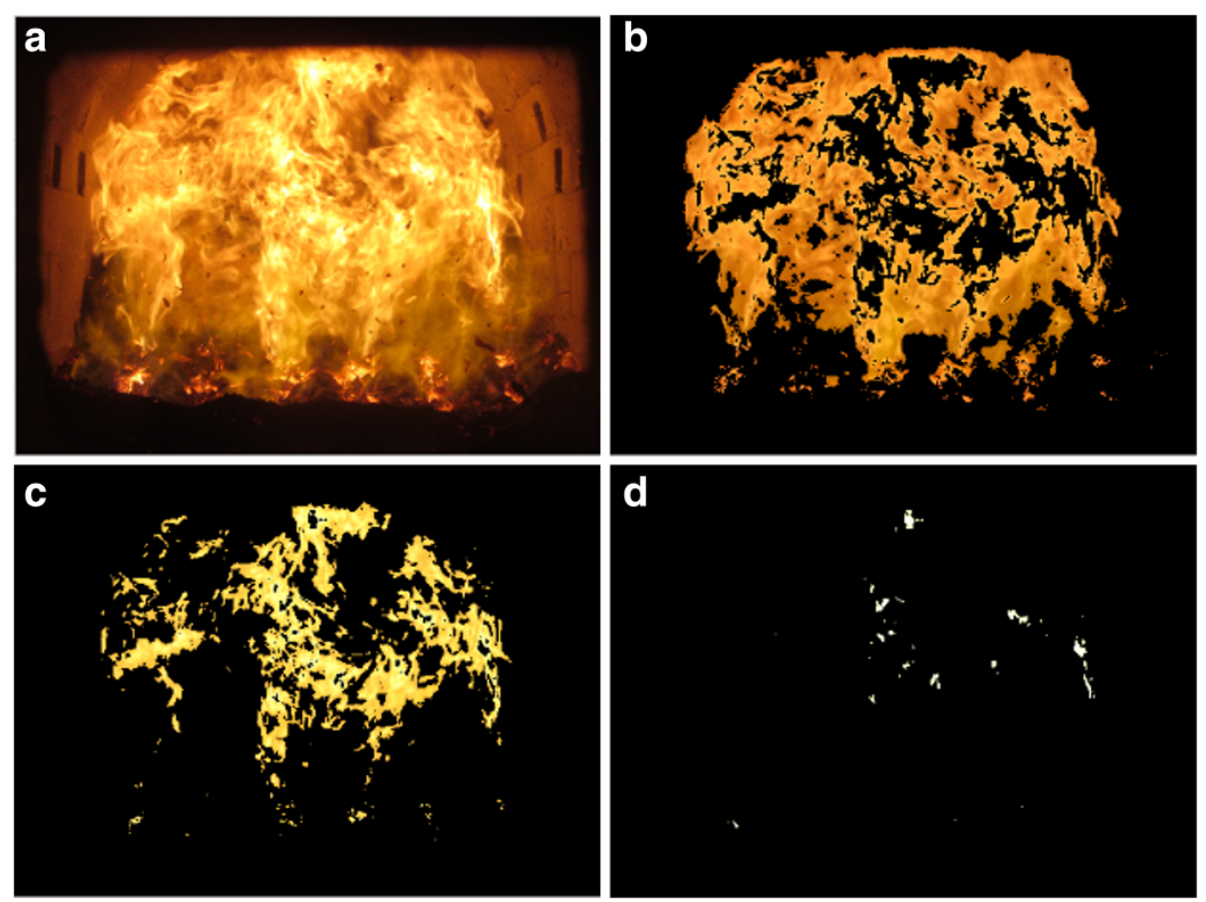

Figure 5 Clustering of the image of a flame with respect to temperature. Above is the figure of an original flame image. The figures below show the low-, medium-, and high-temperature surfaces of the original image that were identified using cluster analysis of the color information in the image.

For substrate feed optimization, the objective function is defined to be a weighted sum of the net income (income from selling electrical and thermal energy minus the operating energy and substrate costs) and a number of operating stability constraints. The constraints considered include a limit on the $\mathrm{pH}$ value inside the digesters, a maximum VFA/TA value, and a minimum methane fraction of $50 \%$ inside the produced biogas. All constraints are normalized between 0 and 1 , with 0 indicating that the according constraint is not violated. The available amount of substrates is a further constraint which is implemented as a hard constraint for the input variables.

In Figure 6, a result of a substrate feed optimization scenario with respect to the objective function is visualized. In this scenario, the substrate feed of a full-scale agricultural biogas plant of $750 \mathrm{~kW}$ was optimized using PSO. The standard substrate feed of this plant is a mixture of maize silage and manure. Optimization showed that using grass silage as additional substrate, the same amount of methane can be produced but with less substrate costs.

The results showed that it is possible to find the optimal substrate feed for long-term operation of biogas plants and therefore to optimize biogas plant operation. This method does offline substrate feed optimization because the substrate feed is not corrected automatically in case a mismatch between predicted and real plant behavior occurs. Thus, a consequent continuation of this method is to implement an online substrate feed optimization as is presented in the following section.

To achieve online substrate feed optimization, model predictive control (MPC) techniques [21] for a closedloop control of the substrate feed are used, which utilize the model of the process to predict the optimal sequence of substrate feeds. As the anaerobic digestion process is nonlinear, nonlinear MPC (NMPC) is used to exploit the full knowledge available of the process to forecast optimal substrate feeds. In Figure 7, a standard NMPC control loop is sketched, which visualizes the developed optimal substrate feed control. In comparison to $\mathrm{MPC}$, the analytical optimization method used in MPC is replaced by a CI method, such as PSO or GA, due to the nonlinearity of the developed model of the biogas plant. The state estimator in Figure 7 is needed to estimate the current state of the biogas plant $\hat{\mathbf{X}}_{k}$ out of the current substrate feed $\mathbf{u}_{\mathrm{opt}, k}$ and the measurements $\mathbf{y}_{k}$ available. In this case, this is done using a supervised learning algorithm which learns a pattern between measurements and the corresponding state of the biogas plant [22].

Figure 8 shows the evolution of the objective value for an algorithm test of the NMPC over a simulated period of 100 days. Here, the controlled biogas plant is the same model as is used by the NMPC for prediction. The individual points (squares) plotted in Figure 8 represent the 


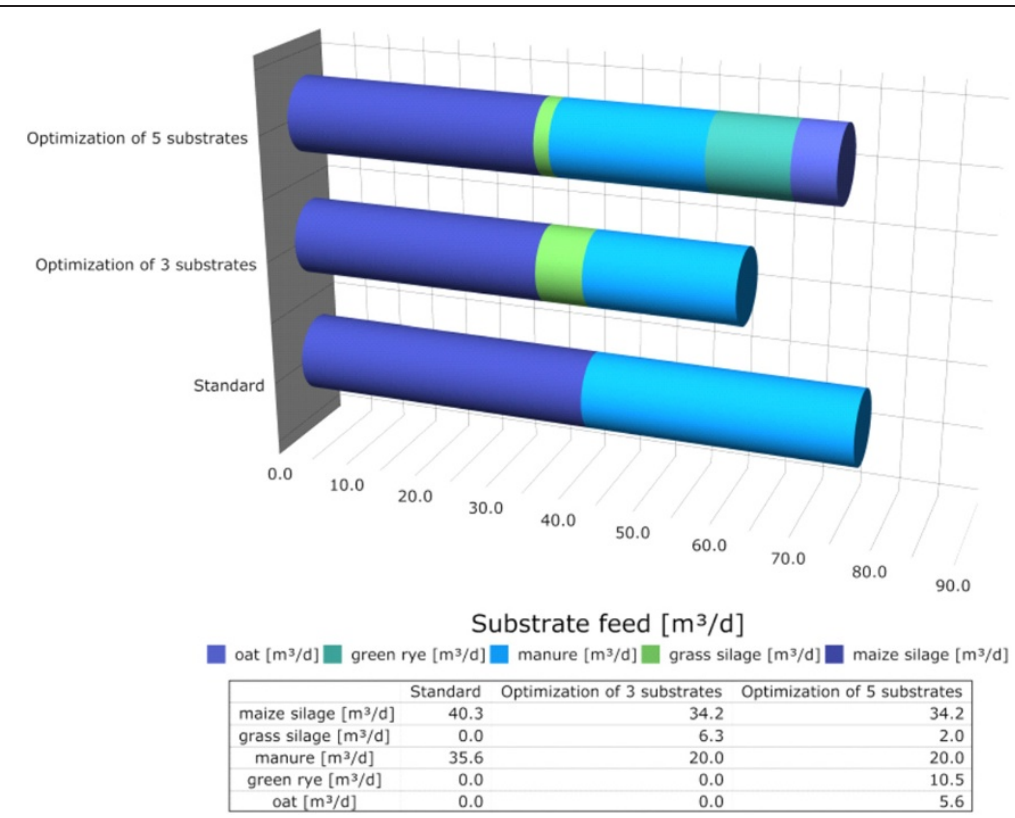

Figure 6 Substrate feed optimization results for a full-scale agricultural biogas plant. Comparison between standard substrate feed and an attempt with three optimized substrates as well as an attempt with five optimized substrates.

simulations performed in the constrained optimization problem over the prediction horizon. The simulations are started at a substrate feed composed out of $30 \mathrm{~m}^{3} /$ day maize silage and $15 \mathrm{~m}^{3}$ /day manure. It can be seen that the plant's operating state was poor in the beginning (warm colors) but improved as the controller drove the plant towards an optimal solution (cold colors). In practical terms, the improvement from 'bad' to 'excellent' achieved by the NMPC represents an additional gain of about $550 € /$ day for the biogas plant operator [23].

\section{Cooperating software agent system for the control of} distributed nonlinear systems

To control a sewer system, every storm water tank (SWT) as well as the wastewater treatment plant (WWTP) is represented by a single software agent. The system follows an economic approach where storm water tanks act as competitors on a virtual water market. The advantage of this approach, compared to conventional control strategies, is the minor configuration effort and the comfortable expandability of this system by achieving the same and

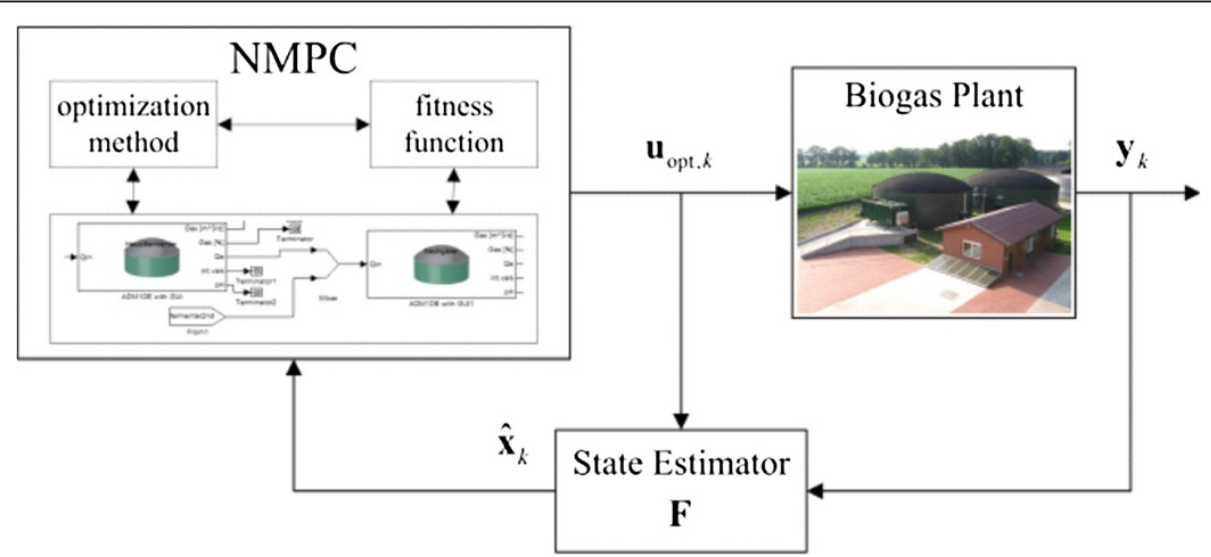

Figure 7 NMPC feedback control system for optimal substrate feed control of biogas plants. The figure visualizes the control loop containing the NMPC, the state estimator, and the controlled biogas plant. 


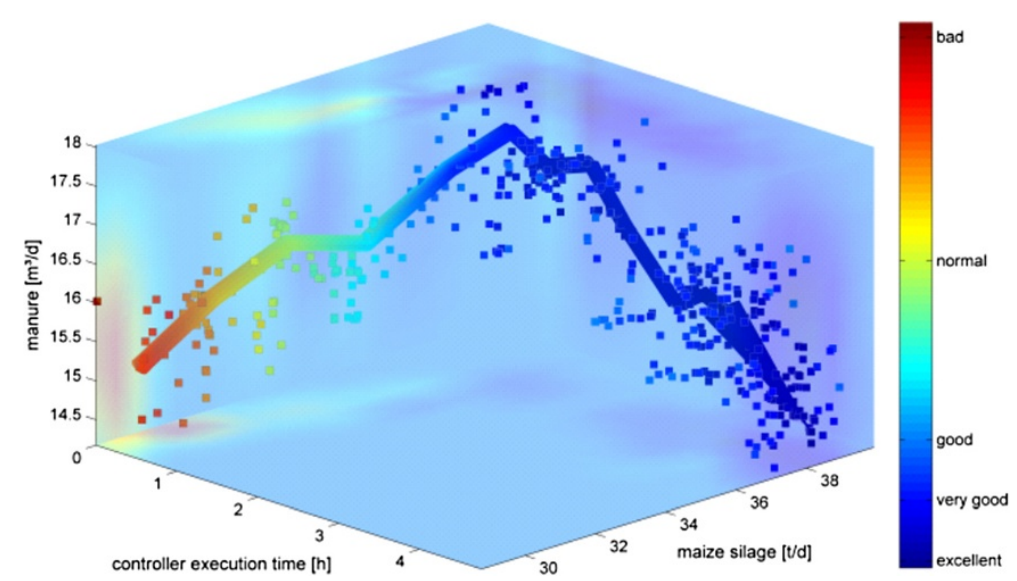

Figure 8 Overall performance of the evaluated substrate mixes during optimal control. Figure 8 shows the evolution of the objective value for an algorithm test of the NMPC over a simulated period of 100 days. The individual points (squares) plotted in Figure 8 represent the simulations performed in the constrained optimization problem over the prediction horizon.

better results than conventional control strategies. It achieves this by self-organization and cooperation of the agents in the system. Control strategies like minimizing water pollution of affiliated rivers or optimizing the wastewater treatment plant inflow can easily be adjusted just by adjusting one or two parameters of the software agents.

The controller development for sewer systems based on classical control concepts is usually complicated and inflexible. A control system is often tailored to a specific sewerage network and is rarely - if ever - immediately transferrable to other sewerage networks. Often, the development of a new control system is necessary. In the following, an alternative approach to conventional control strategies is proposed.

\section{The free market approach}

Comparing functions of the main components in a sewage system with those of the market, we see a variety of analogies. In both systems, there is a product, a supplier of the storage, a buyer of the product, and a common transport system. These similarities lead to the assumption that comparable system rules also exist. This resulted in the approach to depict the behavior of a market with a multi-agent system. The individual participants in the market are represented by software agents with different functions.

First, there are active components such as buyers, sellers, and intermediaries who are actively involved in the trade. Secondly, there are passive components such as the merchandise, product storage, and transport system, which are influenced by the active components. The product or commodity that is traded on the water market of the agent is wastewater. It has a price and can, depending on the regulatory approach, possess a quality that affects the selling price. The transport system for traded goods is the sewer system. This transport system has certain constraints, which are caused by the physical conditions of sewer systems. The buyer represents the wastewater treatment plant. $\mathrm{He}$ is the only real buyer in the water market. The buyer determines the optimal inflow rate to the treatment plant and tries to buy it. Its aim is to optimize the treatment process with the regard to effluent quality and energy consumption.

The seller represents the storm water tank with the respective catchment areas. To any storm water tank, a specific catchment area is assigned which produces wastewater. The seller has no influence on the amount of wastewater produced which is why its aim is to sell the maximum amount of wastewater possible without losing the water by exceeding the storage capacity. The reservoirs of the SWT correspond here to the stored amount of water. The starting price for the sale bid price is calculated in a price function which includes the stored amount of water, the current discharge volume, the priority of the seller agent, and if intended, the quality of the wastewater. The seller is restricted to offer only a part of its water in a round of selling. This ensures that it cannot sell its entire water volume in the first round. The seller, however, has the goal to sell as much as possible. If he is undercut by other sellers, he reduces his selling price.

The negotiator is the instance between buyer and seller. In physical terms, we can assign a sewerage strand with its SWT to the middleman. The middleman takes the purchasing bid of the buyer and tries to get the required amount from the sellers as cheap as possible. To do this, he collects all offers in each round and determines the best deal. The seller with the lowest price may sell its quantity. The trade rounds are completed as soon as the required amount has been purchased or no more goods 


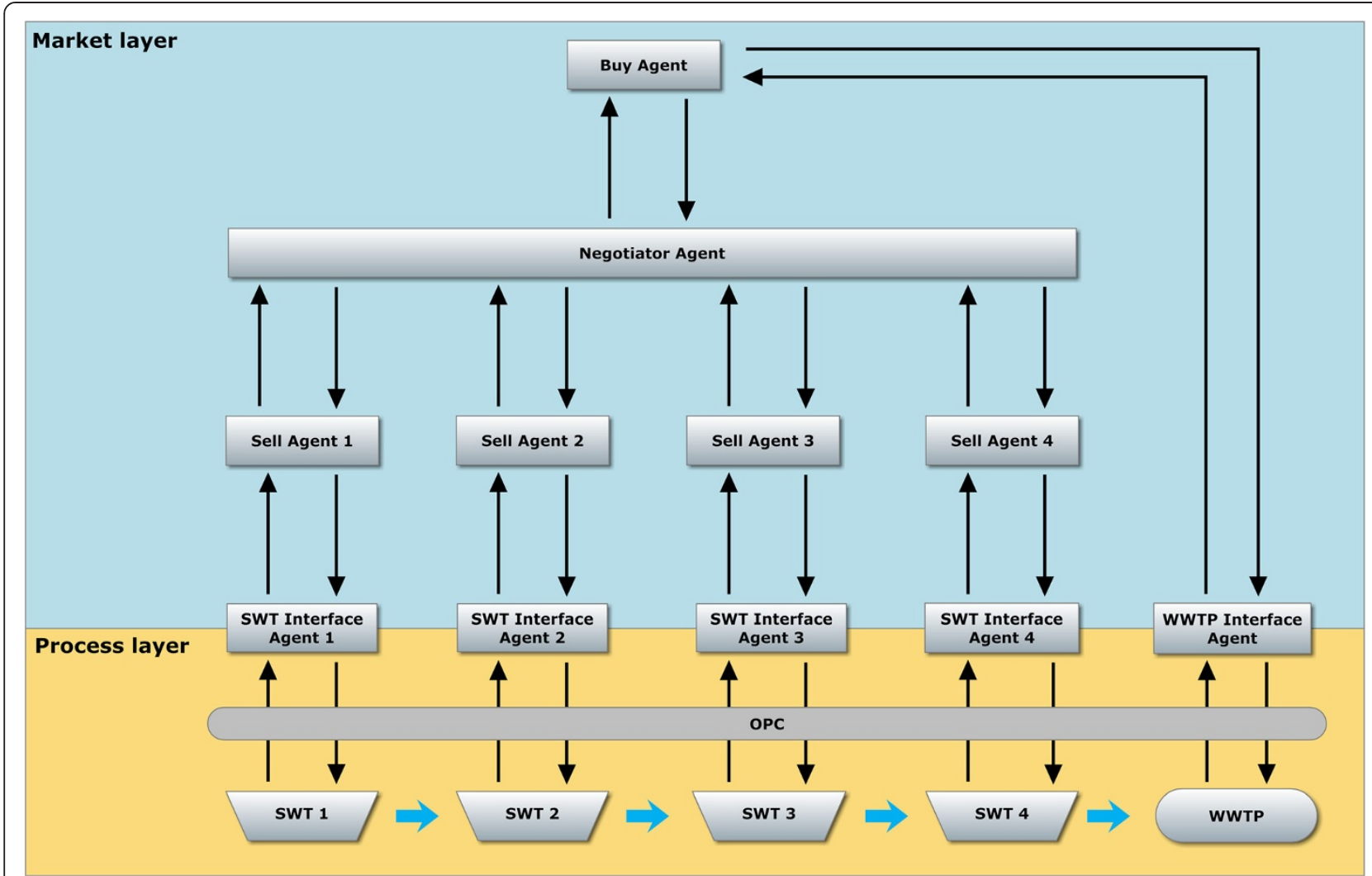

Figure 9 Interaction between the different agents and the physical process. The seller (storm water tank) could offer water over a price. The negotiator is the instance between the buyer and seller. He collects all sell offers in each round and determines the best deal. The seller with the lowest price may sell its quantity.

are offered. Figure 9 shows the principle interaction between the different agents and the physical process.

This method has been tested in sewerage systems for the first time and shows great promise. In the future, this technology will probably dominate the control and coordination in smart energy distribution grids.

\section{Conclusions}

In this paper, five different applications emerging at the intersection of computational intelligence and environmental engineering were presented. It could be shown that (1) using substrate pretreatment-specific biogas production of a biogas plant can be improved and that (2) machine learning methods can learn to measure important process values out of UV/vis and MIR spectroscopic measurements. (3) A simulation model of a biogas plant can predict its behavior and can be used to optimize and control plant operation. (4) Cluster analysis is able to analyze the incineration process of a pellet heater, and (5) multi-agent systems can control sewerage systems. The obtained results show that environmental systems can be substantially improved by intelligent data analysis, optimization, and control methods. Most of the presented technologies have a clear advantage because of a low cost-benefit ratio. Compared to classical methods of mechanical or chemical engineering, the installation of an intelligent data analysis and automation system requires only a fraction of the costs that are necessary to, e.g., enlarge the volume of an anaerobic digester or a storm water retention tank. And, the results in terms of process improvement are similar.

\section{Endnotes}

${ }^{a}$ GECO $>C$ - Gummersbach Environmental Computing Center - Information: www.gecoc.de.

${ }^{\mathbf{b}}$ :metabolon, the Centre of Research and Expertise of Cologne University of Applied Sciences. The project is co-financed by the European Regional Development Fund (ERDF).

${ }^{\mathbf{c}}$ Funded by the BMWi in line with the ZIM program under the support code KF2137807AK1.

Competing interests

The authors declare that they have no competing interests.

\section{Authors' contributions}

$M B$ initialized and financed all projects conducted in this publication, presented the work at CAPE conference, and wrote parts of the manuscript DG worked on feed control of biogas plants, supported the data analysis of the UV/vis spectrometer, and drafted most parts of the manuscript. OT has 
written about and carried out the image analysis of the pellet-heating process and the multi-agent control of the sewerage system. CW did the biogas plant benchmarking, analyzed the UV/Vis and MIR data, and studied feed optimization of biogas plants. Furthermore, he wrote parts of the manuscript and proofread it completely. All authors read and approved the final manuscript.

\section{Authors' information}

Prof. Dr.-Ing. Michael Bongards (1954) studied chemical engineering. After an industrial career, he became professor at the Cologne University of Applied Sciences in 1991. In his scientific work, he focuses on the development of automation solutions for environmental technologies and for the use of regenerative energy. In 2005, he founded the Gummersbach Environmental Computing Center (GECOC) as a university-internal research group. His present work is using methods of computational intelligence to improve simulation systems and to optimize operation of wastewater treatment plants and of anaerobic digesters. Together with his research team, he participated in numerous European and international research projects, especially in simulation, instrumentation, and control of biogas plants.

Daniel Gaida

In 2003, Daniel Gaida started to study Automation Systems at Cologne University of Applied Sciences where he received his BSc in 2006. Right after, he started to study Electrical Engineering and Information Technology at University of Applied Sciences in Düsseldorf. Having obtained his MSc degree in 2009, he returned to Cologne University of Applied Sciences to work in the research group GECO C. In 2009, he also started his PhD studies at Leiden University, the Netherlands, focusing on optimal biogas plant feed control.

Oliver Trauer

Oliver Trauer started his study of Computer Engineering at the Cologne University of Applied Sciences (CUAS) back in 1999 and received his Diploma in 2005. Right after his degree, he started to work as a research associate at the research group GECO C. Parallel to his research work, he started to study Information Engineering part time at CUAS, where he received his M. Sc. in 2010. His main field of work is the development of complex, Cl-aided control systems for industry tasks. During his research work, he developed an industry-rewarded multi-agent system for the control of sewerage systems. Christian Wolf

Christian Wolf studied Electronic Engineering majoring in Automation and Industrial IT at Cologne University of Applied Sciences from 2001 till 2006 graduating as Dipl.-Ing. (FH). Subsequently, he began to work at GECO C under the supervision of Prof. Bongards as a postgraduate researcher in the fields of instrumentation, optimization, and control of wastewater treatment and renewable energy from anaerobic digestion. In parallel to his work at GECO C, he started a part-time Ph.D. in electronic engineering about 'Simulation, optimization and instrumentation of agricultural biogas plants' at the National University of Ireland Maynooth graduating in 2013. Currently, Christian Wolf works as a PostDoc researcher and junior lecturer at Cologne University of Applied Sciences in Gummersbach.

\section{Acknowledgements}

Our colleague Nicole Ley is gratefully acknowledged for editing the final manuscript and managing this publication. Furthermore, all students at Cologne University of Applied Sciences who contributed in one of the projects are acknowledged for their contribution.

\section{Received: 12 August 2014 Accepted: 14 August 2014}

\section{W.}

\section{References}

1. Chen SH, Jakeman AJ, Norton JP (2008) Artificial Intelligence techniques: an introduction to their use for modelling environmental systems. Math Comput Simul 78(2-3):379-400

2. Mellit A, Kalogirou SA, Hontoria L, Shaari S (2009) Artificial intelligence techniques for sizing photovoltaic systems: a review. Renew Sustain Energy Rev 13(2):406-419

3. Jiang Z (2009) Computational intelligence techniques for a smart electric grid of the future. Lect Notes Comput Sci - Adv Neural Networks 5551:1191-1201
4. Carlsson M, Lagerkvist A, Morgan-Sagastume F (2012) The effects of substrate pre-treatment on anaerobic digestion systems: a review. Waste Manag 32 (9):1634-1650

5. Duda RO, Hart PE, Stork DG (2000) Pattern Classification, 2nd edn. Wiley-Interscience, New York, p 654

6. Kusiak A, Zhang Z, Verma A (2013) Prediction, operations, and condition monitoring in wind energy. Energy In Press

7. Maier HR, Jain A, Dandy GC, Sudheer KP (2010) Methods used for the development of neural networks for the prediction of water resource variables in river systems: current status and future directions. Environ Model Softw 25(8):891-909

8. Cinar D, Kayakutlu G, Daim T (2010) Development of future energy scenarios with intelligent algorithms: case of hydro in Turkey. Energy 35 (4):1724-1729

9. Crisci C, Ghattas B, Perera G (2012) A review of supervised machine learning algorithms and their applications to ecological data. Ecol Modell 240:113-122

10. Di Piazza A, Di Piazza MC, Ragusa A, Vitale G (2011) Environmental data processing by clustering methods for energy forecast and planning. Renew Energy 36(3):1063-1074

11. Batstone DJ, Keller J, Angelidaki I, Kalyuzhnyi SV, Pavlostathis SG, Rozzi A, Sanders WTM, Siegrist H, Vavilin VA (2002) Anaerobic Digestion Model No.1 (ADM1), 1st edn. IWA Publishing, London, p 92

12. Clerc M (2006) Particle Swarm Optimization (ISTE). Wiley-ISTE, London, p 243

13. Mitchell M (1998) An Introduction to Genetic Algorithms (Complex Adaptive Systems), A Bradford Book., p 221

14. Barreteau O, Bousquet F, Millier C, Weber J (2004) Suitability of multi-agent simulations to study irrigated system viability: application to case studies in the Senegal River Valley. Agric Syst 80(3):255-275

15. Campo PC, Mendoza GA, Guizol P, Villanueva TR, Bousquet F (2009) Exploring management strategies for community-based forests using multiagent systems: a case study in Palawan, Philippines. J Environ Manage 90 (11):3607-3615

16. Purnomo H, Mendoza GA, Prabhu R, Yasmi Y (2005) Developing multi-stakeholder forest management scenarios: a multi-agent system simulation approach applied in Indonesia. For Policy Econ 7(4):475-491

17. Sausse C, Le Bail M, Lecroart B, Remy B, Messéan A (2013) How to manage the coexistence between genetically modified and conventional crops in grain and oilseed collection areas? Elaboration of scenarios using role playing games. Land Use Policy 30(1):719-729

18. Souchère V, Millair L, Echeverria J, Bousquet F, Le Page C, Etienne M (2010) Co-constructing with stakeholders a role-playing game to initiate collective management of erosive runoff risks at the watershed scale. Environ Model Softw 25(11):1359-1370

19. Wolf C, Gaida D, Stuhlsatz A, Ludwig T, McLoone S, Bongards M (2011) Predicting organic acid concentration from UV/Vis spectrometry measurements - a comparison of machine learning techniques. Trans Inst Meas Control 35(1):5-15

20. Otsu N (1979) A threshold selection method from Gray-level histograms. IEEE Trans Syst Man Cybern 9(1):62-66

21. Findeisen R, Imsland L, Allgöwer F, Foss BA (2003) State and output feedback nonlinear model predictive control: an overview. Eur J Control 9 (2-3):190-206

22. Gaida D, Wolf C, Meyer C, Stuhlsatz A, Lippel J, Bäck T, Bongards M, McLoone S (2012) State estimation for anaerobic digesters using the ADM1. Water Sci Technol 66(5):1088-1095

23. Gaida D, Wolf C, Bäck T, Bongards M (2012) 2012 20th Mediterranean Conference on Control \& Automation (MED)., pp 652-657

doi:10.1186/s13705-014-0019-3

Cite this article as: Bongards et al.: Intelligent automation and IT for the optimization of renewable energy and wastewater treatment processes. Energy, Sustainability and Society 2014 4:19. 